\title{
Regulation of interstitial excitatory amino acid concentrations after cortical contusion injury
}

\author{
Marie E. Rose ${ }^{\mathrm{a}, \mathrm{e}}$, Michele B. Huerbin ${ }^{\mathrm{a}, \mathrm{e}}$, John Melick ${ }^{\mathrm{b}, \mathrm{d}, \mathrm{e}}$, Donald W. Marion ${ }^{\mathrm{c}, \mathrm{e}}$, \\ Alan M. Palmer ${ }^{\mathrm{f}}$, Joanne K. Schiding ${ }^{\mathrm{b}, \mathrm{d}, \mathrm{e}}$, Patrick M. Kochanek ${ }^{\mathrm{b}, \mathrm{d}, \mathrm{e}}$, \\ Steven H. Graham ${ }^{\mathrm{a}, \mathrm{e}, \mathrm{g}, *}$ \\ ${ }^{a}$ Department of Neurology, 526 South BST, University of Pittsburgh, Pittsburgh, PA 15213, USA \\ ${ }^{\mathrm{b}}$ Department of Anesthesiology and Critical Care Medicine, University of Pittsburgh, Pittsburgh, PA, USA \\ ${ }^{\mathrm{c}}$ Department of Neurosurgery, University of Pittsburgh, Pittsburgh, PA, USA \\ ${ }^{\mathrm{d}}$ Safar Center for Resuscitation Research, University of Pittsburgh, Pittsburgh, PA, USA \\ ${ }^{\mathrm{e}}$ Brain Trauma Research Center, University of Pittsburgh, Pittsburgh, PA, USA \\ ${ }^{\mathrm{f}}$ Cerebrus Ltd., Wokingham, UK \\ ${ }^{\mathrm{g}}$ Geriatric Research Educational and Clinical Center, Veterans Affairs Pittsburgh Health System, Pittsburgh, PA, USA
}

Accepted 22 November 2001

\begin{abstract}
Increases in brain interstitial excitatory amino acid $\left(\mathrm{EAA}_{\mathrm{I}}\right)$ concentrations after ischemia are ameliorated by use-dependent Na+ channel antagonists and by supplementing interstitial glucose, but the regulation of EAA $\mathrm{I}_{\mathrm{I}}$ after traumatic brain injury (TBI) is unknown. We studied the regulation of $\mathrm{EAA}_{\mathrm{I}}$ after TBI using the controlled cortical impact model in rats. To monitor changes in EAA , microdialysis probes were placed in the cortex adjacent to the contusion and in the ipsilateral hippocampus. Significant increases in dialysate EAA $_{\mathrm{I}}$ after TBI were found compared to levels measured in sham controls. Treatment with the use-dependent Na+ channel antagonist $619 \mathrm{C} 89$ (30 mg/kg i.v.) did not significantly decrease dialysate glutamate compared to vehicle controls in hippocampus $(10.4 \pm 2.4$ vs. $11.9 \pm 1.6 \mu \mathrm{M})$, but there was significant decrease in dialysate glutamate in cortex after $619 \mathrm{C} 89$ treatment $(19.3 \pm 3$ vs. 12.6 $\pm 1.1 \mu \mathrm{M} P<0.05)$. Addition of $30 \mathrm{mM}$ glucose to the dialysate, a treatment that decreases EAA $\mathrm{I}_{\mathrm{I}}$ after ischemia, had no significant effect upon dialysate glutamate after TBI in cortex $(20.0 \pm 4.9$ vs. $11.7 \pm 3.4 \mu \mathrm{M})$ or in hippocampus $(10.9 \pm 2.0 \mathrm{vs}$. $8.9 \pm 2.4 \mu \mathrm{M})$. These results suggest that neither increased release of EAAs due to $\mathrm{Na}+$ channel-mediated depolarization nor failure of glutamate reuptake due to glucose deprivation can explain the majority of the increase in EAA following TBI. (C) 2002 Published by Elsevier Science B.V.
\end{abstract}

Theme: Disorders of the nervous system

Topic: Trauma

Keywords: Brain microdialysis; Glucose; Glutamate; Traumatic brain injury

\section{Introduction}

Excitatory amino acids (EAAs) may mediate, at least in part, neuronal damage in traumatic brain injury (TBI). Excessive concentrations of EAAs such as glutamate are highly toxic to neurons in culture [3]. Microdialysis studies

\footnotetext{
*Corresponding author. Geriatric Research Educational and Clinical Center, VA Pittsburgh Health System, Pittsburgh, PA 15240, USA. Tel.: +1-412-648-3299; fax: +1-412-648-1239.

E-mail address: sgra@pitt.edu (S.H. Graham).
}

have shown that increased concentrations of brain interstitial excitatory amino acids ([EAA $\left.{ }_{\mathrm{I}}\right]$ ) exist in animal models of TBI $[6,17]$. Activation of EAA receptors results in increased glucose metabolism after TBI [9]. Furthermore, administration of agents that inhibit both NMDA and non-NMDA excitatory amino acid receptors improve behavioral outcome in such models $[1,6,8,14,20]$.

While increases in $\left[\mathrm{EAA}_{\mathrm{I}}\right]$ as monitored by microdialysis have been demonstrated to occur after TBI in rats, very little is known about the underlying mechanisms. In the current study we address two possible mechanisms 
that have been shown to contribute to increased $\left[\mathrm{EAA}_{\mathrm{I}}\right]$ in ischemia: depolarization of neurons mediated by $\mathrm{Na}+$ channels, and decreased supply of glucose. Use-dependent inhibitors of brain (Type II) $\mathrm{Na}+$ channels prevent prolonged, pathological opening of neuronal sodium channels and thus ameliorate depolarization of neurons $[5,11,13]$. Such agents, including BW1003, 619C89, and phenytoin, have been shown to ameliorate increases in $\left[\mathrm{EAA}_{\mathrm{I}}\right]$ during cerebral ischemia $[7,12,15,19]$. In ischemia, deprivation of both oxygen and glucose supplies occurs. If high concentrations of glucose are added to the microdialysate, increases in $\left[\mathrm{EAA}_{\mathrm{I}}\right]$ are almost completely inhibited even with complete ischemia (by permanent cardiac arrest) [24]. These data imply that increased $\left[\mathrm{EAA}_{\mathrm{I}}\right]$ is due to failure of glutamate reuptake mechanisms that can be fueled by anaerobic glucose metabolism.

Much less is known about what determines $\left[\mathrm{EAA}_{\mathrm{I}}\right]$ after trauma. In the current study, we determine the changes in post-TBI $\left[\mathrm{EAA}_{\mathrm{I}}\right]$ as a result of treatment with the usedependent sodium channel antagonist 619C89 and as a result of supplementation of the local glucose supply by the addition of $30 \mathrm{mM}$ glucose to the microdialysate.

\section{Materials and methods}

\subsection{Surgical procedure}

This protocol was approved by the Animal Care and Use Committee of the University of Pittsburgh. Male SpragueDawley rats (Harlen Sprague Dawley, Indianapolis, IN, USA), $250-350 \mathrm{~g}$, with free access to food and water prior to experiments, were anesthetized with $4 \%$ isoflurane (IsoFlo, Abbot Laboratories, North Chicago, IL, USA) and $\mathrm{N}_{2} \mathrm{O}-\mathrm{O}_{2}$ (70:30), and intubated with a $14 \mathrm{G}$ angiocath. Isoflurane was lowered to $2 \%$ for surgery ( $1 \%$ for maintenance, and rats ventilated (Harvard Small Animal Ventilator, Harvard Apparatus, South Natick, MA, USA) with a tidal volume of $1 \mathrm{cc} / 100 \mathrm{~g}$, adjusting the rate to yield $\mathrm{PaO}_{2}>90 \mathrm{mmHg}$ and $\mathrm{PaCO}_{2} 35-45 \mathrm{mmHg}$. The right femoral artery was cannulated using PE50 tubing for continuous blood pressure monitoring, blood gases, hematocrit and glucose.

Rats were placed in a stereotactic device (Kopf Instruments, Tujunga, CA, USA) and prepared for trauma by exposure of the left parietal cortex via craniotomy. A brain temperature probe (Physitemp IT23, Physitemp Instruments, Clifton, NJ, USA) was placed in the ipsilateral frontal cortex via a burr hole, and both brain and rectal temperatures were maintained at $37 \pm 0.5^{\circ} \mathrm{C}$ by use of a heating pad and lamp.

\subsection{Controlled cortical impact}

Rats underwent lateral cortical contusion injury (CCI) using a vertical impactor as previously described [10]. A stroke-constrained pneumatic cylinder (Bimba Manufacturing, Monee, IL, USA), mounted on an adjustable crossbar, was positioned to provide vertical impact with a $5-\mathrm{mm}$ rounded impactor tip. Velocity and duration of impact were determined using an integrated accelerometer and computer with experimental parameters of $4.0 \pm 0.1 \mathrm{~m} / \mathrm{s}$ and $40-50 \mathrm{~ms}$, respectively. Depth of penetration of $2.5 \mathrm{~mm}$ was controlled by zeroing the piston to the surface of the dura prior to microdialysis probe insertion.

\subsection{Microdialysis}

Artificial cerebral spinal fluid (CSF) was prepared as follows: $141 \mathrm{mM} \mathrm{NaCl}, 5 \mathrm{mM} \mathrm{KCl}, 1.3 \mathrm{mM} \mathrm{CaCl}_{2}, 1.3$ $\mathrm{mM} \mathrm{MgCl}_{2}$. Microdialysis probes (CMA 12, CMA Microdialysis, Acton, MA, USA) were loaded with CSF and pumped at $2 \mu \mathrm{l} / \mathrm{min}$ (Harvard Pump 22, Harvard Apparatus) $30 \mathrm{~min}$ prior to implantation. Sample collection was adjusted to allow for dead space in probes and exit tubing. Two microdialysis probes were mounted on a micromanipulator arm and lowered into the ipsilateral hippocampus and cortex at the following coordinates (from Bregma): hippocampus-AP 4.3, lateral 2.0, depth 3.5 $\mathrm{mm}$; cortex-AP 4.3, lateral 5.0, depth $4.5 \mathrm{~mm}$. Samples were collected every $10 \mathrm{~min}$ for an equilibration period of $1 \mathrm{~h}$. Probes were removed from the hippocampus and cortex for trauma and replaced within 2 min of CCI as previously described [17]. Dialysate samples were again collected every $10 \mathrm{~min}$ for $1 \mathrm{~h}$ post injury, with tubes stored at $-70{ }^{\circ} \mathrm{C}$ until high-performnace liquid chromatography (HPLC) analysis. At the completion of the experiment, the animals were euthanized with an overdose of pentobarbital and the brains removed and inspected to confirm probe placement position.

\subsection{Sham versus CCI groups}

Rats were divided into two groups ( $n=4-8$ per group), $\mathrm{CCI}$ and sham, with all surgeries and physiologic parameters identical except for the omission of CCI. Microdialysis probes were prepared and loaded as described above, collecting dialysate $1 \mathrm{~h}$ prior to trauma. CCI animals underwent trauma while sham animals had probes removed for $2 \mathrm{~min}$ with no CCI. Probes for both groups were reinserted and dialysate collected every $10 \mathrm{~min}$ for $1 \mathrm{~h}$ after trauma.

\subsection{Glucose microdialysis treatment group}

Artificial CSF was prepared as above with either 0 or 30 $\mathrm{mM}$ glucose. Animals were divided into two groups $(n=$ $10-15$ per group): CCI with $0 \mathrm{mM}$ glucose CSF, and CCI with $30 \mathrm{mM}$ glucose CSF. Probes were inserted and trauma performed exactly as described above. 


\subsection{C89 treatment group}

Rats underwent the same microdialysis procedure as described above. They were divided into two groups $(n=8$ per group): vehicle control (double-distilled water), and 619C89 (30 mg/kg). This dose was the maximally effective dose in previous temporary focal ischemia experiments. Furthermore, doses higher than $30 \mathrm{mg} / \mathrm{kg}$ were found to produce hypotension, which could confound the interpretation of these experiments [7]. The $30 \mathrm{mg} / \mathrm{kg}$ dose was found to afford behavioral protection in the rat fluid percussion model [22]. Vehicle or drug was administered i.v. over $1 \mathrm{~min}$ after the equilibration period, with drug concentration adjusted to yield a final injection volume of $1 \mathrm{ml}$. Five minutes after injection, CCI was performed and samples collected for $1 \mathrm{~h}$ post trauma.

\section{7. $H P L C$}

The concentration of glutamate was determined by HPLC with scanning fluorescence detection (Waters 470 scanning fluorescence detector, excitation wavelength 334 $\mathrm{nm}$, emission wavelength $424 \mathrm{~nm}$ ). $25 \mu \mathrm{l}$ samples were diluted 1:1 with $0.01 \mathrm{M} \mathrm{HCl}$, mixed with $80 \mu \mathrm{l} o$ phthaldialdehyde-mercaptoethanol (Sigma) and injected into the column after $1 \mathrm{~min}$ (Waters 715 Ultra Wisp). A reversed-phase column and guard column $(100 \times 4.6 \mathrm{~mm}$ I.D. and $15 \times 4.6 \mathrm{~mm}$ I.D., respectively; $\mathrm{C}_{18}$ Microsorb, Varian, Sugarland, TX, USA), packed with $3 \mu \mathrm{m}$ octadecylsilane particles, were contained in an axial compression unit (Dynamax, Rainin Instrument) at $30{ }^{\circ} \mathrm{C}$. Mobile phase was comprised of $50 \mathrm{mM} o$-phosphoric acid, $50 \mu \mathrm{M}$ EDTA, pH 5.7 with $\mathrm{NaOH}$ (all chemicals: Sigma). This mobile phase was mixed with methanol (Optima, Fisher Scientific, Pittsburgh, PA, USA) in a ratio of $(90: 10, \mathrm{v} / \mathrm{v})$ for solvent A and (10:90, v/v) for solvent B. Samples were injected using a curvilinear gradient, changing the proportion of solvent A from $80 \%$ to $10 \%$ over 16 min, returning to $80 \%$ over $5 \mathrm{~min}$. Peaks were identified by comparing retention times with those of standards, and quantified using Maxima 820 software (Dynamic Solutions, Ventura, CA, USA) after calibration to standard curves. Amino acid concentrations are expressed as the actual dialysate concentrations and were not corrected for recovery.

\subsection{Statistical analysis}

The a priori hypothesis was that the mean peak dialysate concentrations in samples obtained immediately after trauma would differ for treatment groups versus controls. Accordingly, this comparison was made with a $t$-test. The significance of comparisons at other time points was assessed using analysis of variance (ANOVA) with Bonferroni post hoc testing. The minimal level of significance accepted was $P<0.05$.

\section{Results}

Figs. 1 and 2 and illustrate the effects of various treatments upon dialysate glutamate concentrations in hippocampus and cortex after CCI. Since the probe was removed from the brain just prior to and reinserted just after trauma, changes in dialysate glutamate were first studied in traumatized rats and rats that were subjected to
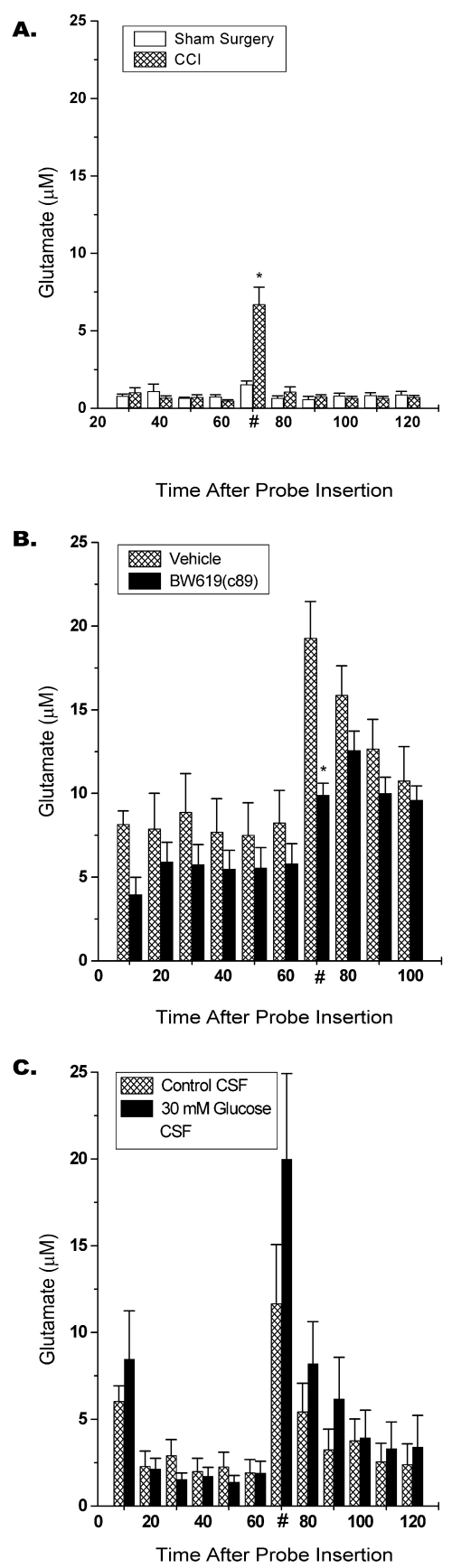

Fig. 1. Time course of glutamate dialysate concentrations $(\mu \mathrm{M})$ from cortex prior to and after controlled cortical impact. \#: CCI occurs immediately prior to this sample. (A) CCI vs. sham. (B) 619C89 30 $\mathrm{mg} / \mathrm{kg}$ i.v. $5 \mathrm{~min}$ pre-CCI vs. vehicle. (C) $30 \mathrm{mM}$ glucose dialysate vs. 0 $\mathrm{mM}$ glucose dialysate. ${ }^{*} P<0.05$. 

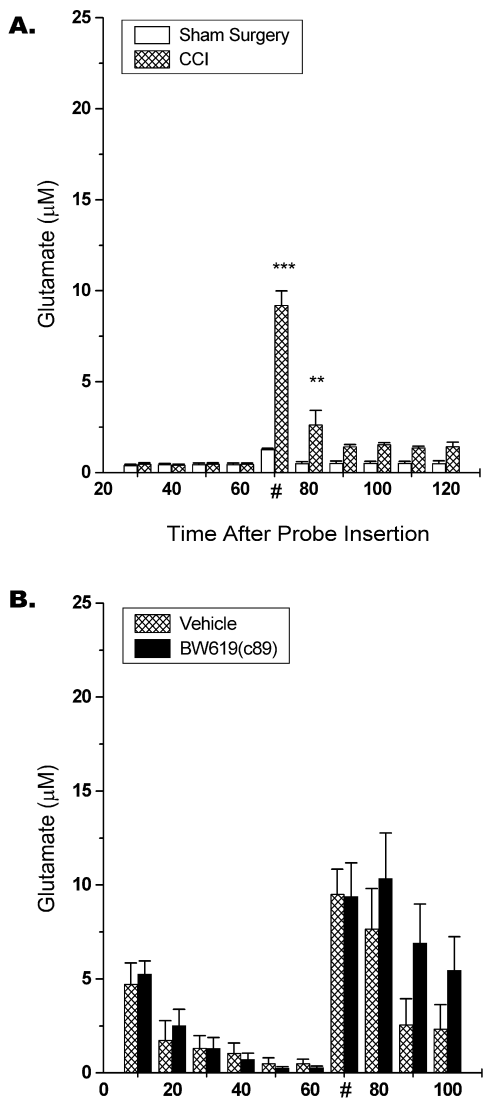

Time After Probe Insertion

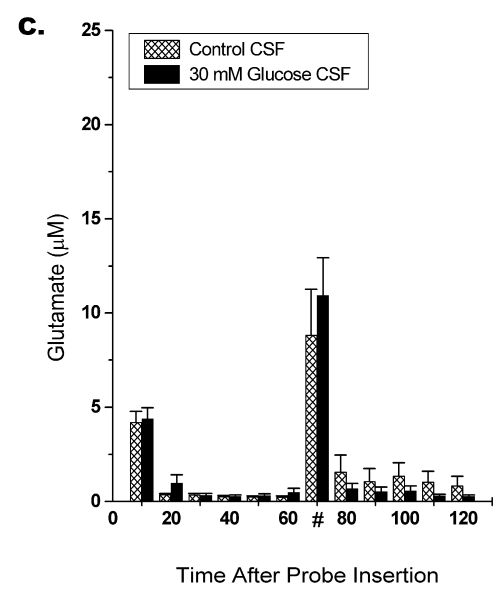

Fig. 2. Time course of glutamate dialysate concentrations $(\mu \mathrm{M})$ from hippocampus prior to and after controlled cortical impact. \#: CCI occurs immediately prior to this sample. (A) CCI vs. sham. (B) 619C89 30 $\mathrm{mg} / \mathrm{kg}$ i.v. $5 \mathrm{~min}$ pre-CCI vs. vehicle. (C) $30 \mathrm{mM}$ glucose dialysate vs. 0 $\mathrm{mM}$ glucose dialysate. $* * P<0.01$, $* * * P<0.001$.

the sham operation, including probe removal and reinsertion. There was a slight trend toward increased microdialysis glutamate in the sample immediately after probe reinsertion in the sham-operated group, but these changes were not significantly different from baseline concentrations. However, in keeping with previous studies, the rats subjected to CCI showed a significant increase in microdialysate glutamate concentrations in the sample immediately after probe reinsertion. There were also increases in the excitatory amino acids aspartate and glycine in the CCI group (Table 1).

Treatment with the use-dependent $\mathrm{Na}+$ channel antagonist 619C89 produced a significant effect on microdialysis glutamate in cortex, but not in hippocampus (Figs. 1B and 2B). There was also a significant effect on dialysate aspartate in cortex. Paradoxically, there was an increase in dialysate glycine in the 619C89 treatment group (Table 1). The magnitudes of these changes are less compared to the near total suppression of ischemia-induced changes in dialysate glutamate and other EAAs by 619 C 89 treatment [7].

There was no significant effect of altering the glucose concentration in the dialysate upon microdialysate glutamate in either cortex (Fig. 1C) or hippocampus (Fig. 2C). Paradoxically, there was an increase in dialysate aspartate in cortical samples obtained in the $30 \mathrm{mM}$ glucose dialysate group, although there was no significant effect of altering dialysate glucose upon any dialysate amino acid measured in hippocampus (Table 1). Thus, in contrast to cerebral ischemia, dialysate glucose does not have a significant effect on interstitial EAA concentrations after CCI.

\section{Discussion}

The major findings of this study are: (1) increased interstitial concentrations of the EAA glutamate occur in cortex after CCI. Increased concentrations also occur in hippocampus, a site remote to the contusion but synaptically connected to cortex. (2) Treatment with the use-dependent glutamate inhibitor 619C89 decreased concentrations of dialysate glutamate in cortex, but not in hippocampus. (3) Altering the concentration of microdialysate glucose produced no effect on $\left[\mathrm{EAA}_{\mathrm{I}}\right]$ in either region.

The CCI model of traumatic brain injury produces a circumscribed contusion in rat cortex; however, this injury produces additional effects at sites distant from the contusion. The hippocampus is one area where CCI results in death of rare pyramidal neurons in CA3 and additional loss of $10-25 \%$ of the dentate granule cells [4]. The impact produces a pressure wave, which may mediate injury at these distant sites. Furthermore, cortical neurons also project to the hippocampus, so cortical injury may alter synaptic activity in hippocampus. In this study, microdialysate glutamate and other neurotransmitter amino acids are increased in hippocampus, demonstrating that CCI has effects at this distant site.

Ischemia-induced increases in $\left[\mathrm{EAA}_{\mathrm{I}}\right]$ can be ameliorated by supplying additional glucose to the probe surrounds via $30 \mathrm{mM}$ glucose in the dialysate. This treatment almost completely blocked increases in $\left[\mathrm{EAA}_{\mathrm{I}}\right]$ after complete ischemia induced by cardiac arrest [24]. Further- 
Table 1

Amino acid peak concentrations $(\mu \mathrm{M})$ at time of controlled cortical impact

\begin{tabular}{|c|c|c|c|c|}
\hline Group & Aspartate & Serine & Glutamine & Glycine \\
\hline \multicolumn{5}{|l|}{ Cortex } \\
\hline SHAM CCI & $0.44 \pm 0.04$ & $5.77 \pm 0.30$ & $6.29 \pm 1.78$ & $7.48 \pm 0.65$ \\
\hline CCI & $1.99 \pm 0.59 *$ & $5.75 \pm 0.90$ & $7.58 \pm 0.91$ & $9.30 \pm 0.51 *$ \\
\hline $0 \mathrm{mM}$ Glucose CSF & $4.68 \pm 1.73$ & $9.59 \pm 2.02$ & $19.80 \pm 4.00$ & $9.53 \pm 2.66$ \\
\hline $30 \mathrm{mM}$ Glucose CSF & $11.43 \pm 3.34^{*}$ & $18.26 \pm 3.35^{*}$ & $23.32 \pm 3.24$ & $25.44 \pm 10.70$ \\
\hline Vehicle & $9.57 \pm 1.45$ & $11.71 \pm 0.96$ & $19.75 \pm 1.79$ & $16.67 \pm 1.73$ \\
\hline $30 \mathrm{mg} / \mathrm{kg}$ 619C89 & $3.56 \pm 0.67 * *$ & $8.82 \pm 0.37 *$ & $12.30 \pm 1.92 *$ & $24.31 \pm 3.27 *$ \\
\hline \multicolumn{5}{|l|}{ Hippocampus } \\
\hline SHAM CCI & $0.96 \pm 0.59$ & $5.11 \pm 0.08$ & $3.71 \pm 0.24$ & $7.19 \pm 0.25$ \\
\hline $\mathrm{CCI}$ & $2.92 \pm 0.55^{*}$ & $7.16 \pm 0.54 * *$ & $7.10 \pm 0.84 * *$ & $10.50 \pm 0.22 * * *$ \\
\hline $0 \mathrm{mM}$ Glucose CSF & $3.48 \pm 1.66$ & $9.77 \pm 1.97$ & $18.13 \pm 2.28$ & $6.48 \pm 1.49$ \\
\hline $30 \mathrm{mM}$ Glucose CSF & $4.53 \pm 2.13$ & $9.69 \pm 0.82$ & $19.27 \pm 1.97$ & $6.57 \pm 1.61$ \\
\hline Vehicle & $2.71 \pm 0.90$ & $8.69 \pm 0.94$ & $17.64 \pm 1.08$ & $10.70 \pm 1.63$ \\
\hline $30 \mathrm{mg} / \mathrm{kg} 619 \mathrm{C} 89$ & $3.52 \pm 0.90$ & $6.99 \pm 0.73$ & $11.00 \pm 0.82 * * *$ & $9.46 \pm 2.71$ \\
\hline
\end{tabular}

Data are mean \pm S.E.M. CCI: Controlled cortical impact; CSF: cerebral spinal fluid. $* P<0.05, * * P<.01, * * * P<0.001$ vs. control group.

more, the effect of glucose on $\left[\mathrm{EAA}_{\mathrm{I}}\right]$ could be reversed by the addition of a glutamate transporter inhibitor, threobeta-hydroxyaspartate, to the dialysate. This suggests that the reduction in $\left[\mathrm{EAA}_{\mathrm{I}}\right]$ fueled by microdialysate glucose is the result of anerobically fueled glutamate reuptake. Swanson et al. have shown that astrocyte glutamate uptake continues at the normal rate even when mitochondrial respiration is blocked by fluoroacetate and other such agents $[23,25]$. Thus, the failure of glial reuptake due to deprivation of glucose supplies may be a major factor accounting for glial reuptake in ischemia.

In the CCI model of TBI, however, there was no significant effect of $30 \mathrm{mM}$ glucose in the dialysate upon the EAA glutamate in either cortex or hippocampus. Although there are decreases in cerebral blood flow after trauma, these decreases do not reach the threshold for significant ischemia, except in the center of the contusion itself [10]. Therefore, one would not expect a lack of glucose to be the primary factor in this TBI model. While the degree of blood flow deprivation is not as severe as in ischemia models, there are areas of increased glucose utilization after TBI [9]. Thus, it is possible that ischemia could exist in these regions and that a shortfall of available glucose leads to increased $\left[\mathrm{EAA}_{\mathrm{I}}\right]$ after TBI. These current results do not support this latter hypothesis. Unlike ischemia, supplying glucose via the dialysate cannot completely abrogate the increase in $\left[\mathrm{EAA}_{\mathrm{I}}\right]$ after CCI. Hence, a substantial portion of the transient increases in $\left[\mathrm{EAA}_{\mathrm{I}}\right]$ are unrelated to glucose supplies and therefore must be due to other mechanisms.

A second mechanism that has been proposed as a cause of increased $\left[\mathrm{EAA}_{\mathrm{I}}\right]$ in cerebral ischemia is depolarization of neurons. Drugs such as 619C89 are anticonvulsants that inhibit $\mathrm{Na}+$ channels within the axon and thus inhibit neuronal depolarization. Unlike tetrotoxin, which irreversibly blocks these $\mathrm{Na}+$ channels, 619C89's action is use- dependent: it acts only on open channels to promote closing. Thus, the drug inhibits the prolonged opening of $\mathrm{Na}+$ channels that occurs in pathologic states including ischemia. 619C89 has been shown to significantly reduce $\left[\mathrm{EAA}_{\mathrm{I}}\right]$ in cortex during ischemia $[7,12]$.

There is also evidence that glutamate released from synapses plays a role in hippocampal pathology after CCI. Lesioning CA3 with kainate prior to CCI prevented the trauma-induced increases in glucose utilization [26]. Treatments with drugs that prevent the increase of $\left[\mathrm{EAA}_{\mathrm{I}}\right]$ by inhibiting $\mathrm{Na}+$ channels also have neuroprotective effects in TBI models. Treatment with 619C89 has also been shown to improve behavioral outcome and reduce neuronal loss in hippocampus after fluid percussion injury in rats [22]. In the current study, treatment with the use-dependent $\mathrm{Na}+$ channel inhibitor 619C89 did significantly reduce peak glutamate microdialysate concentrations in cortex, but not in hippocampus. These results suggest that depolarization of neurons after TBI is not the major determinant of increased $\left[\mathrm{EAA}_{\mathrm{I}}\right]$ after trauma, at least in cortex adjacent to the contusion. However, unlike the effect in ischemia models, treatment with 619C89 only partially reduced the increase in $\left[\mathrm{EEA}_{\mathrm{I}}\right]$ after CCI. No changes were detected in hippocampus, suggesting that other mechanisms (perhaps the direct mechanical effects on neurons) may account for the increased $\left[\mathrm{EAA}_{\mathrm{I}}\right]$ in this region. A possible explanation for this is suggested by the results of Bullock et al., who found similar increases in neurotransmitters in human microdialysate samples obtained after TBI [2]. They propose that the increase in $\left[\mathrm{EAA}_{\mathrm{I}}\right]$ after trauma is the result of nonspecific release from damaged membranes rather than release from synaptic stores. This hypothesis is supported by the lack of a robust effect of 619C89 treatment upon [EAA ${ }_{\mathrm{I}}$ ] after trauma.

There are several limitations to these findings. First, measurement of $\left[\mathrm{EAA}_{\mathrm{I}}\right]$ by microdialysis has many po- 
tential artifacts, not the least of which is the trauma induced by the probe itself. The inclusion of a shamoperated group where the probe was inserted, removed, then reinserted was used to control for these potential confounding factors. Furthermore, microdialysis detects changes in interstitial amino acids, but may be insensitive to changes within the synapse, where excitatory amino acid receptors are found. For example, while there is abundant evidence that excitotoxicity contributes to neuronal death in epilepsy, increases in $\left[\mathrm{EAA}_{\mathrm{I}}\right]$ are not detectable by microdialysis during status epilepticus [21]. Thus, the failure of $619 \mathrm{C} 89$ to reduce $\left[\mathrm{EAA}_{\mathrm{I}}\right]$ in the hippocampus does not preclude an effect limited to the synapse. Therefore, 619C89 could still have therapeutic effects on neurons outside the contusion independent of changes in $\left[\mathrm{EAA}_{\mathrm{I}}\right]$ detectable by microdialysis. The rat CCI model mimics some aspects of human head injury but is a less severe and more circumscribed injury than those of many patients with severe head injury. Furthermore, there are many differences in the changes in [EAAI] observed in severely head-injured patients who have been studied with microdialysis compared to rodents subjected to CCI [27]. Finally, as in any in vivo pharmacological experiment, the action of 619C89 in this experiment could be mediated by mechanisms other than those intended. For example, drugs such as NMDA antagonists that have a cytoprotective effect could prevent leakage of glutamate into the interstitial space [18]. Such an effect could also explain the discrepancy between 619C89's effect in cortex, where there is extensive cell death, and in hippocampus, where almost all cells survive. Other studies are required to confirm these observations and address these issues.

Despite these limitations, the current experiments provide additional insight into the mechanisms that govern increased $\left[\mathrm{EAA}_{\mathrm{I}}\right]$ after TBI. Interstitial glucose, like hypothermia [16], is a potent inhibitor of the increase in $\left[\mathrm{EAA}_{\mathrm{I}}\right]$ after ischemia, but has little or no effect in the CCI model of TBI. The use-dependent $\mathrm{Na}+$-channel antagonist 619C89 did ameliorate the increase in $\left[\mathrm{EAA}_{\mathrm{I}}\right]$ after CCI in cortex, suggesting that a component of increased $\left[\mathrm{EAA}_{\mathrm{I}}\right]$ results from neuronal depolarization in cortex.

\section{Acknowledgements}

This work was supported by NIH P50 NS30318. The authors thank Pat Strickler for secretarial assistance and Carol Culver for editing.

\section{References}

[1] H. Bernert, L. Tursi, Traumatic brain damage prevented by the non- $N$-methyl-D-aspartate antagonist 2,3-dihydroxy-6-nitro-7-sulfamoylbenzo[f] quinoxaline, Proc. Natl. Acad. Sci. USA 93 (1996) $5235-5240$
[2] R. Bullock, A. Zauner, J.J. Woodward, J. Myseros, S.C. Choi, J.D. Ward, A. Marmarou, H.F. Young, Factors affecting excitatory amino acid release following severe human head injury, J. Neurosurg. 89 (1998) 507-518.

[3] D.W. Choi, G.M. Maulucci, A.R. Kriegstein, Glutamate neurotoxicity in cortical cell culture, J. Neurosci. 7 (1987) 357-368.

[4] R.S.B. Clark, P.M. Kochanek, C.E. Dixon, M.Z. Chen, D.W. Marion, S. Heineman, S.T. DeKosky, S.H. Graham, Early neuropathologic effects of mild or moderate hypoxemia after controlled cortical impact injury in rats, J. Neurotrauma 14 (1997) 179-189.

[5] M.D. Edwards, M.J. Leach, G. Lees, Molecular mode of action of the neuroprotective agent BW619C89, Anesthesiology 87 (1994) 851, (Abstract).

[6] A.I. Faden, P. Demediuk, S.S. Panter, R. Vink, The role of excitatory amino acids and NMDA receptors in traumatic brain injury, Science 244 (1989) 798-800.

[7] S.H. Graham, J. Chen, J. Lan, M.J. Leach, R.P. Simon, Neuroprotective effects of a use-dependent blocker of voltage-dependent sodium channels, BW619C89, in rat middle cerebral artery occlusion, J. Pharmacol. Exp. Ther. 269 (1994) 854-859.

[8] R.L. Hayes, L.W. Jenkins, B.G. Lyeth, R.L. Balster, S.E. Robinson, G.L. Clifton, J.F. Stubbins, H.F. Young, Pretreatment with phencyclidine, an $\mathrm{N}$-methyl-D-aspartate antagonist, attenuates longterm behavioral deficits in the rat produced by traumatic brain injury, J. Neurotrauma 5 (1988) 259-274.

[9] T. Kawamata, Y. Katayama, D.A. Hovda, A. Yoshino, D.P. Becker, Administration of excitatory amino acid antagonists via microdialysis attenuates the increase in glucose utilization seen following concussive brain injury, J. Cereb. Blood Flow Metab. 12 (1992) $12-24$.

[10] P.M. Kochanek, D.W. Marion, W. Zhang, J.K. Schiding, M. White, A.M. Palmer, R.S. Clark, M.E. O’Malley, S.D. Styren, C. Ho et al., Severe controlled cortical impact in rats: assessment of cerebral edema, blood flow, and contusion volume, J. Neurotrauma 12 (1995) 1015-1025.

[11] M.J. Leach, C.M. Marden, A.A. Miller, Pharmacological studies on lamotrigine, a novel potential antiepileptic drug: II. Neurochemical studies on the mechanism of action, Epilepsia 27 (1986) 490-497.

[12] M.J. Leach, J.H. Swan, D. Eisenthal, M. Dopson, M. Nobbs, BW619C89, a glutamate release inhibitor, protects against focal cerebral ischemic damage, Stroke 24 (1993) 1063-1067.

[13] G. Lees, M.J. Leach, Studies on the mechanism of action of the novel anticonvulsant lamotrigine (Lamictal) using primary neurological cultures from rat cortex, Brain Res. 612 (1993) 190-199.

[14] T.K. McIntosh, R. Vink, H. Soares, R. Hayes, R. Simon, Effects of the $N$-methyl-D-aspartate receptor blocker MK-801 on neurologic function after experimental brain injury, J. Neurotrauma 6 (1989) 247-259.

[15] B.S. Meldrum, J.H. Swan, M.J. Leach, M.H. Millan, R. Gwinn, K. Kadota, S.H. Graham, J. Chen, R.P. Simon, Reduction of glutamate release and protection against ischemic brain damage by $\mathrm{BW}$ 1003C87, Brain Res. 593 (1992) 1-6.

[16] A.M. Palmer, D.W. Marion, M.L. Botscheller, E.E. Redd, Therapeutic hypothermia is cytoprotective without attenuating the traumatic brain injury-induced elevations in interstitial concentrations of aspartate and glutamate, J. Neurotrauma 10 (1993) 363-372.

[17] A.M. Palmer, D.W. Marion, M.L. Botscheller, P.E. Swedlow, S.D. Styren, S.T. DeKosky, Traumatic brain injury-induced excitotoxicity assessed in a controlled cortical impact model, J. Neurochem. 61 (1993) 2015-2024.

[18] S.S. Panter, A.I. Faden, Pretreatment with NMDA antagonists limits release of excitatory amino acids following traumatic brain injury, Neurosci. Lett. 136 (1992) 165-168.

[19] J. Rataud, F. Debarnot, V. Mary, J. Pratt, J.M. Stutzmann, Comparative study of voltage-sensitive sodium channel blockers in focal ischaemia and electric convulsions in rodents, Neurosci. Lett. 172 (1994) 19-23. 
[20] Y. Shapira, G. Yadid, S. Cotev, A. Niska, E. Shohami, Protective effect of MK801 in experimental brain injury, J. Neurotrauma 7 (1990) 131-139.

[21] S.E. Smith, D. Lekieffre, P. Sowinski, B.S. Meldrum, Cerebroprotective effect of Bw619C89 after focal or global cerebral ischaemia in the rat, Neuroreport 4 (1993) 1339-1342.

[22] F.Y. Sun, A.I. Faden, Neuroprotective effects of 619C89, a usedependent sodium channel blocker, in rat traumatic brain injury, Brain Res. 673 (1995) 133-140.

[23] R.A. Swanson, Astrocyte glutamate uptake during chemical hypoxia in vitro, Neurosci. Lett. 147 (1992) 143-146.

[24] R.A. Swanson, J. Chen, S.H. Graham, Glucose can fuel glutamate uptake in ischemic brain, J. Cereb. Blood Flow Metab. 14 (1994) $1-6$.

[25] R.A. Swanson, S.H. Graham, Fluorocitrate and fluoroacetate effects on astrocyte metabolism in vitro, Brain Res. 664 (1994) 94-100.

[26] A. Yoshino, D.A. Hovda, Y. Katayama, T. Kawamata, D.P. Becker, Hippocampal CA3 lesion prevents postconcussive metabolic dysfunction in CA1, J. Cereb. Blood Flow Metab. 12 (1992) 996-1006.

[27] A. Zauner, R. Bullock, A.J. Kuta, J. Woodward, H.F. Young, Glutamate release and cerebral blood flow after severe human head injury, Acta Neurochir. Suppl. 67 (1996) 40-44. 